\title{
AVALIAÇÃO DO PROCESSO DE SEPARAÇÃO DE PLÁSTICOS DESCARTÁVEIS - POLIPROPILENO (PP) E POLIESTIRENO DE ALTO IMPACTO (HIPS) - POR DENSIDADE
}

\author{
R. R. BREVES ${ }^{1}$, P. H. C. VIEIRA ${ }^{1}$, R. P. PEÇANHA ${ }^{2}$ e E. B. A. V. PACHECO ${ }^{3}$ \\ ${ }^{1}$ Graduando de Engenharia Química, Escola de Química / UFRJ \\ ${ }^{2}$ Departamento de Engenharia Química, Escola de Química / UFRJ \\ ${ }^{3}$ Instituto de Macromoléculas Professora Eloisa Mano / UFRJ \\ E-mail para contato: rrbreves@ hotmail.com
}

\begin{abstract}
RESUMO - Polipropileno (PP) e poliestireno de alto impacto (HIPS) são polímeros utilizados na fabricação de copos descartáveis. Entretanto, após o uso, estes se acumulam em aterros sanitários, com riscos ambientais e sociais. A reciclagem é uma solução para a destinação sustentável dos materiais pós-consumo, mas requer uma separação prévia dos materiais, sendo este o maior empecilho à sua implantação industrial. Neste trabalho, estudou-se a separação de misturas de PP e HIPS obtidas de copos pós-consumo, que foram lavados, secos e moídos originando flakes de formato essencialmente plano. A diferença de densidade dos materiais possibilitou a separação dos flakes por gravidade em equipamento protótipo de bancada do tipo batelada, especialmente desenvolvido para este fim. Foram testadas misturas dos materiais nas seguintes proporções ponderais de PP/HIPS: 90/10, 70/30, 50/50, 30/70 e 10/90. Obteve-se um produto de fundo de HIPS com contaminação média inferior a $8 \%$ de PP e um produto de topo de PP com contaminação média de $22 \%$ de HIPS. Concluiu-se que a separação dos polímeros é tecnicamente viável pelo método proposto. Sugerem-se estudos em outras escalas e em equipamento contínuo
\end{abstract}

\section{INTRODUÇÃO}

Atualmente, os plásticos têm uso extremamente diversificado sendo bastante úteis à população. Praticamente todos os setores da sociedade usam artefatos feitos a partir de plástico (têxtil, automobilístico, médico - farmacêutico, construção civil e outros). A indústria dos plásticos está em desenvolvimento constante com o surgimento de tecnologias para atender às novas demandas que surgem a cada dia, e não é surpresa que a produção mundial de plástico tenha chegado aos 288 milhões de toneladas em 2012 (PLASTIC EUROPE, 2013).

No entanto, o lado negativo da produção em larga escala e do consumo desenfreado é a geração de uma quantidade enorme de resíduos pós-consumo, tratados como lixo. Ao serem descartados, os resíduos plásticos tornam-se um passivo ambiental, causando problemas da ordem social, ambiental e econômica. Devido a isso, o gerenciamento de plásticos tornou-se um ponto bastante discutido por estudiosos da área e pelos governos. Desse debate, surgiu a Política Nacional de Resíduos Sólidos (PNRS), instituída no Brasil em 2010 pela Lei 12.305 
que trata de resíduos sólidos, dentre eles os plásticos pós-consumo. A Lei estabelece estratégias para o desenvolvimento sustentável e impõe o sistema de logística reversa e reciclagem, além de tratar do ciclo de vida dos produtos e incentivar o reuso e a reciclagem de materiais.

\section{Resinas Poliméricas}

No caso específico do polipropileno (PP) e do poliestireno de alto impacto (HIPS), o consumo e a destinação, geralmente em aterro, não são diferentes do que se observa para os outros plásticos. Eles são utilizados, principalmente, no setor alimentício, bens de consumo e eletrodomésticos. Especificamente para alimentos, são empregados em filmes plásticos, bandejas, recipientes e materiais descartáveis (copos, talheres, pratos).

Muitos dos produtos plásticos de PP e de HIPS, entre eles os de embalagens e bens não duráveis, se tornam resíduo depois de um curto período de uso após a compra ou, em um cenário pior, após um único uso (AL-SALEM et al., 2009). No entanto, estes resíduos possuem considerável valor agregado e são fontes de matérias-primas para a produção de outros produtos ou até de outras substâncias poliméricas. Há ainda uma carência de estudos no desenvolvimento para processos de reciclagem desses dois tipos de plásticos.

\section{Reciclagem de plásticos}

Os plásticos pós-consumo devem atender a ordem de prioridade de redução do consumo, reutilização, reciclagem dos resíduos gerados, tratamento dos rejeitos e, por fim, a disposição em aterros (Lei 12.305, 2010). Quanto a sua reciclagem, existem três formas: mecânica, química ou energética. A primeira consiste na conversão de descartes plásticos em grânulos ou produtos. Os materiais descartados podem ser resíduos oriundos de indústrias ou do pós-consumo, sendo envolvidas somente transformações mecânicas e físicas, como: moagem, fusão e solidificação por resfriamento.

A reciclagem química se refere ao processo que converte polímeros em moléculas menores, em estado líquido ou gasoso, que podem ser reincorporadas na cadeia produtiva de petroquímicos ou intermediários para a fabricação de outras resinas (AL-SALEM et al., 2009). E, finalmente, a reciclagem energética corresponde à combustão dos resíduos e, consequente, recuperação da energia térmica liberada. Nos materiais pós-consumo, existe a necessidade de separação e tratamento prévio dos plásticos presentes antes do processamento mecânico ou químico, pois quanto mais misturados e contaminados forem os resíduos, mais difícil será reciclá-los e obter produto de boa qualidade (AL-SALEM et al., 2009).

Diversos trabalhos sugerem que, depois do reuso, a forma mais ambientalmente correta de reaproveitamento dos plásticos é a reciclagem mecânica (DE OLIVEIRA, 2012). Um aspecto fundamental neste tipo de reciclagem é a qualidade final da resina processada. Embora seja possível reciclar misturas de plásticos, é preferencial que haja só um tipo de plástico. Assim, os processos de separação ganham muita importância por influenciar na capacidade de processamento, propriedades do produto final e preço das resinas recicladas. Para separação de plásticos, existem inúmeras técnicas, manuais ou automáticas, explorando 
as diversas propriedades das resinas plásticas. Dentre elas podem-se citar: separação por gravidade, separação por flotação, separação triboelétrica, dentre outras.

\section{Desenvolvimento do Equipamento de Separação}

O grande entrave à reciclagem de PP e de HIPS é a inexistência de processos eficientes e baratos de separação desses materiais. Existem processos na literatura (Pongstabodee et al., 2008) que descrevem separações de outros plásticos com diferentes densidades por gravidade, mas não para misturas de PP e HIPS, com densidades $0,84 \mathrm{~g} / \mathrm{cm}^{3}$ e $1,03 \mathrm{~g} / \mathrm{cm}^{3}$ respectivamente. A partir do processo clássico de separação por densidade foram identificados os principais problemas ligados à ligados à técnica: baixo índice de automação, dificuldade de coleta do sobrenadante e do produto de fundo, tempo de separação e necessidade de agitação inicial para desagregação do material. Tendo em vista os empecilhos do processo clássico (tanque de separação simples) e as características dos materiais envolvidos, foram desenvolvidos e testados, protótipos em três escalas: 20, 40 e 100 litros.

Inicialmente foi concebido um modelo de 20L de capacidade. Um segundo modelo (40L) foi sugerido visando corrigir os problemas encontrados anteriormente, entupimento e dificuldades para capação do produto final separado. Por sua vez, este também apresentou problemas quanto à configuração estrutural, dificuldade no dimensionamento de tubulações e baixa capacidade instalada para a realização da circulação de água. Por fim, foi necessária uma ampliação na escala do equipamento e um terceiro modelo foi desenvolvido com capacidade de 100L. As adaptações feitas neste modelo conseguiram superar as dificuldades encontradas nos modelos anteriores e obter bons resultados.

\subsection{O Protótipo}

Conforme mostrado esquematicamente na Figura 1, o equipamento consiste de dois reservatórios - tanque de separação superior ("Caixa 1") e tanque de reserva inferior ("Caixa 2") - de maneira que o primeiro alimenta o segundo com o meio de separação utilizado (água) e o segundo alimenta o primeiro com água recirculada. A Caixa 1 é a parte central de todo o sistema e onde ocorre a separação. Neste tanque, é adicionada a amostra a ser separada e as aspersões no topo criam agitação para desagregar o material adicionado e permitir que parte do material decante (HIPS) e outra flutue (PP). Através de uma abertura na parte inferior é criado um fluxo que capta o material decantado, coletado em peneiras, quando este fluxo é interrompido o nível da água aumenta, fazendo com que o sobrenadante seja captado por um duto central e coletado em peneiras. O reservatório inferior serve apenas para captar a água utilizada na separação do tanque superior e alimentar a bomba hidráulica que faz com que a água recircule no sistema. A Tabela 1 identifica as principais partes constituintes do equipamento. 


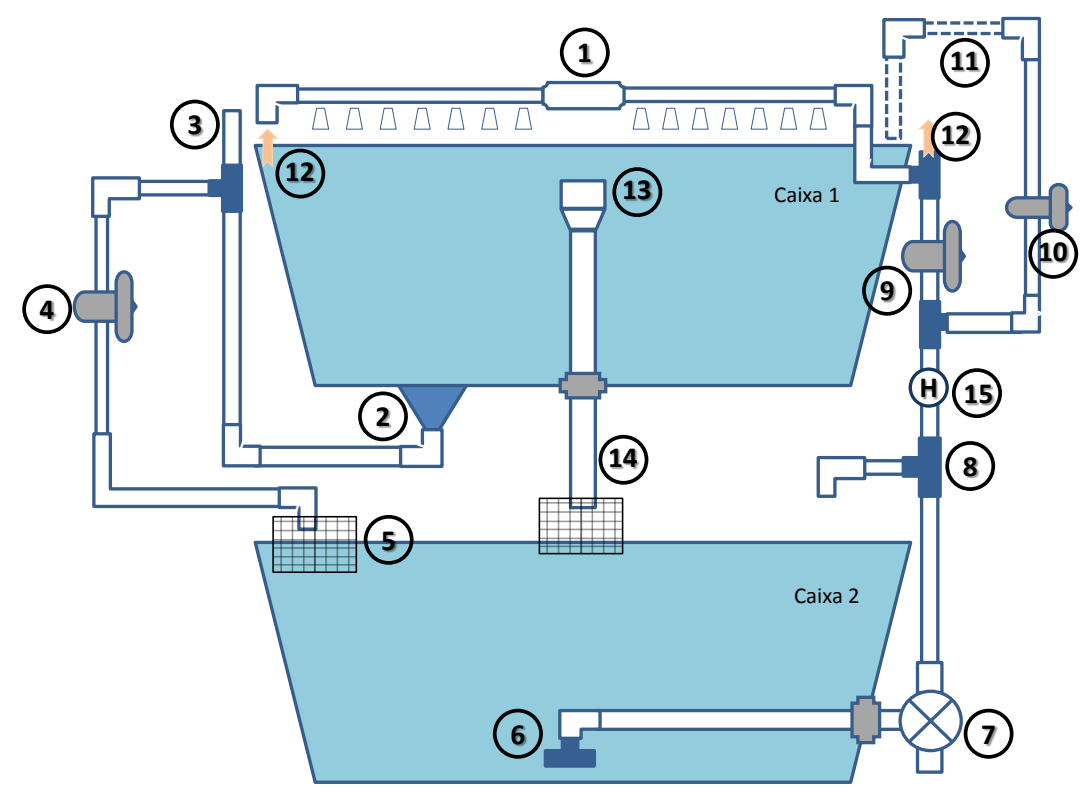

Figura 1 - Representação esquemática do protótipo.

Tabela 1 - Legenda da Figura 1.

\begin{tabular}{|c|c|c|c|}
\hline Número & Elemento & Número & Elemento \\
\hline 1 & Jatos Inclinados ("chafariz") & 9 & Válvula dos jatos superiores \\
\hline 2 & Saída de HIPS & 10 & Válvula da entrada independente \\
\hline 3 & Respiro & 11 & Entrada Independente \\
\hline 4 & Válvula da saída de HIPS & 12 & Entrada dos jatos superiores \\
\hline 5 & Coleta de HIPS & 13 & Cano central \\
\hline 6 & Captação de água para bomba & 14 & Coleta do material superficial \\
\hline 7 & Bomba $(0,5 \mathrm{HP})$ & 15 & Hidrômetro \\
\hline 8 & "By pass" & & \\
\hline
\end{tabular}

\section{Procedimento Experimental}

O conjunto de etapas que compõe o procedimento experimental utilizado é ilustrado na Figura 2.

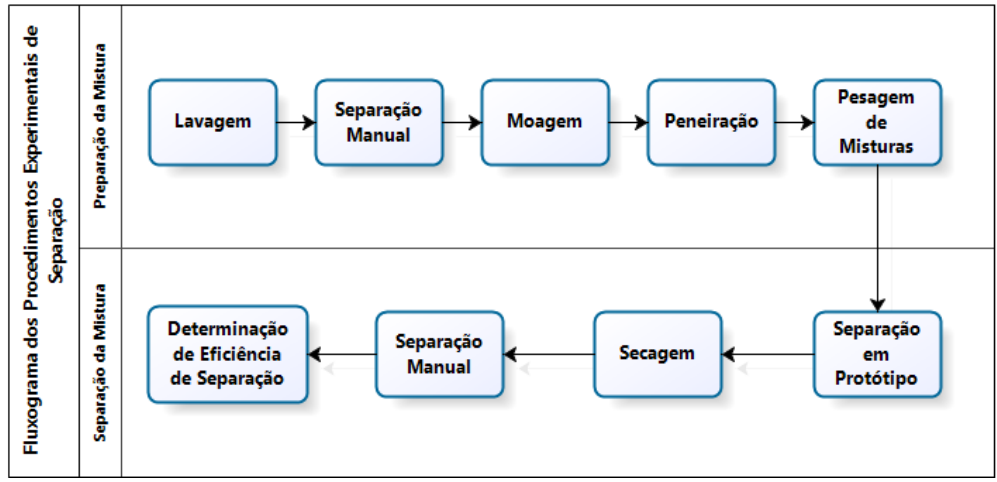

Figura 2 - Procedimento Experimental 


\subsection{Preparação da Mistura}

Lavagem dos copos: os copos pós-consumo foram obtidos a partir do programa de coleta seletiva RECICLA CT, instituído no Centro de Tecnologia (CT) da Universidade Federal do Rio de Janeiro (UFRJ) em 2007. A lavagem foi efetuada manualmente sem o uso de qualquer detergente.

Separação manual: Os copos de PP e HIPS eram facilmente separados já que tinham cores distintas, marrom e branco, respectivamente. Os símbolos de reciclagem existentes no fundo dos copos (marca em alto relevo) garantia a natureza do material.

Moagem: Os copos de cada material foram triturados separadamente, utilizando-se moinho de facas, modelo LP 1003 Primotécnica. As partículas obtidas tinham a forma de flakes essencialmente planos conforme mostra a Figura 3.

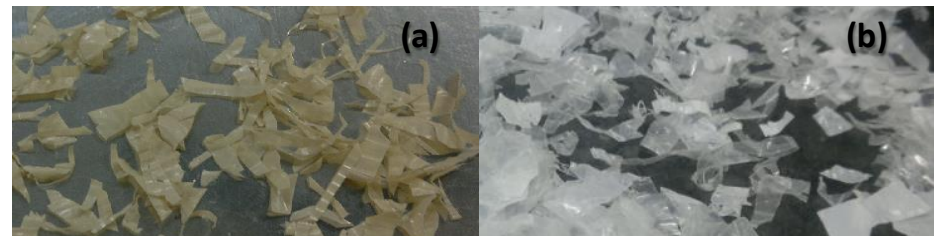

Figura 3 - Fotografias dos flakes coloridos de PP (a) e de HIPS (b) (Imagens por Rafael Breves, em janeiro de 2014).

Peneiração: Experimentos prévios do tipo afunda - flutua em becher, revelaram a presença de partículas muito pequenas, que poderiam interferir no funcionamento da bomba, acumulando-se nas captações de água e aumentando a perda de carga em toda a tubulação ascendente, reduzindo a eficiência do processo. Desta forma, foi necessário peneirar previamente o material para eliminar esses finos. Todavia, o material particulado eliminado por peneiração foi menos de $2 \%$ em massa.

A distribuição de tamanhos das partículas de PP e HIPS assim obtidas, foi estabelecida a partir de fotografias das amostras. As fotos sobre fundo preto fosco, foram feitas com a câmera fotográfica de telefone celular (Nokia N8 - 12MP), conforme mostra a Figura 4. Para efeitos de calibração, uma régua com escalas de $\mathrm{cm}$ e $\mathrm{mm}$ foi fotografada junto. As imagens obtidas foram tratadas com o programa freeware ImageJ (2014) obtendo-se a área projetada de cada partícula, o que permitiu caracterizá-las individualmente pelo diâmetro da esfera de mesma área projetada.

(a)

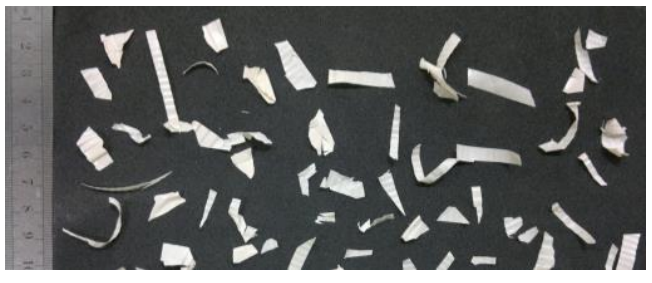

(b)

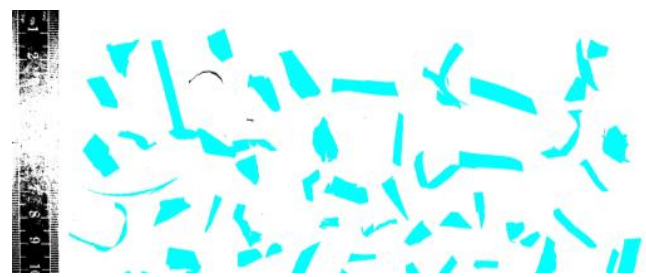

Figura 4 - Exemplo de imagem trata pelo ImageJ.

(a) Fotografia dos flakes (b) imagem tratada com cores invertidas pelo ImageJ. (Fotografia elaborada por Rafael Breves, em janeiro 2014). 
1010 partículas (585 de PP; 425 de HIPS) foram analisadas e para validação do método foram utilizados quadrados e hexágonos de área conhecida, tendo o programa calculado as áreas projetadas com desvios mínimos. A distribuição de tamanhos das partículas de PP e HIPS foram descritas com os modelos de Rosin-Rammler-Bennet (RRB) e Gates-GaudinSchuhmann (GGS). Todavia, apenas para o modelo de RRB os resultados foram satisfatórios com coeficientes de correlação linear superiores a 0,97, conforme demonstrado na Tabela 2.

Tabela 2 - Distribuição de Tamanhos de Mistura de Partículas.

\begin{tabular}{|c|c|c|c|c|c|c|c|}
\hline $\begin{array}{l}\text { Rótulos de } \\
\text { Linha }\end{array}$ & $\begin{array}{c}\text { Soma de } \\
\text { AREA }\left(\mathrm{mm}^{2}\right)\end{array}$ & $\begin{array}{l}\text { Média de } d_{a} \\
\quad(\mathrm{~mm})\end{array}$ & $\begin{array}{l}\text { Contagem } \\
\text { de } d_{a}(m m)\end{array}$ & Xi & Yi & $\begin{array}{c}x \\
\operatorname{Ln}(d a)\end{array}$ & $\begin{array}{c}Y \\
\ln (\ln (1 /(1-y i))\end{array}$ \\
\hline$<45$ & 5.316 & 23 & 1 & $0,10 \%$ & $0,10 \%$ & 3,11 & $-6,92$ \\
\hline $45-65$ & 329.384 & 55 & 31 & $3,07 \%$ & $3,17 \%$ & 4,01 & $-3,44$ \\
\hline $65-85$ & 1.868 .359 & 75 & 98 & $9,70 \%$ & $12,87 \%$ & 4,32 & $-1,98$ \\
\hline 85-105 & 4.502 .447 & 95 & 159 & $15,74 \%$ & $28,61 \%$ & 4,55 & $-1,09$ \\
\hline $105-125$ & 7.671.162 & 115 & 184 & $18,22 \%$ & $46,83 \%$ & 4,74 & $-0,46$ \\
\hline $125-145$ & 11.138 .940 & 135 & 195 & $19,31 \%$ & $66,14 \%$ & 4,91 & 0,08 \\
\hline $145-165$ & 10.706 .842 & 155 & 142 & $14,06 \%$ & $80,20 \%$ & 5,04 & 0,48 \\
\hline $165-185$ & 8.214 .499 & 175 & 86 & $8,51 \%$ & $88,71 \%$ & 5,16 & 0,78 \\
\hline $185-205$ & 5.778 .129 & 195 & 49 & $4,85 \%$ & $93,56 \%$ & 5,27 & 1,01 \\
\hline $205-225$ & 3.884.168 & 215 & 27 & $2,67 \%$ & $96,24 \%$ & 5,37 & 1,19 \\
\hline $225-245$ & 2.410 .873 & 235 & 14 & $1,39 \%$ & $97,62 \%$ & 5,46 & 1,32 \\
\hline $245-265$ & 2.817.562 & 255 & 14 & $1,39 \%$ & $99,01 \%$ & 5,54 & 1,53 \\
\hline $265-285$ & 705.356 & 275 & 3 & $0,30 \%$ & $99,31 \%$ & 5,62 & 1,60 \\
\hline 285-305 & 844.455 & 295 & 3 & $0,30 \%$ & $99,60 \%$ & 5,69 & 1,71 \\
\hline 305-325 & 293.594 & 315 & 1 & 0,00099 & $99,70 \%$ & 5,75 & 1,76 \\
\hline$>325$ & 1.158 .322 & 325 & 3 & 0,00297 & $100,00 \%$ & 5,78 & \\
\hline Total Geral & 62.329 .408 & 132,7555065 & 1010 & $100,00 \%$ & & & \\
\hline
\end{tabular}

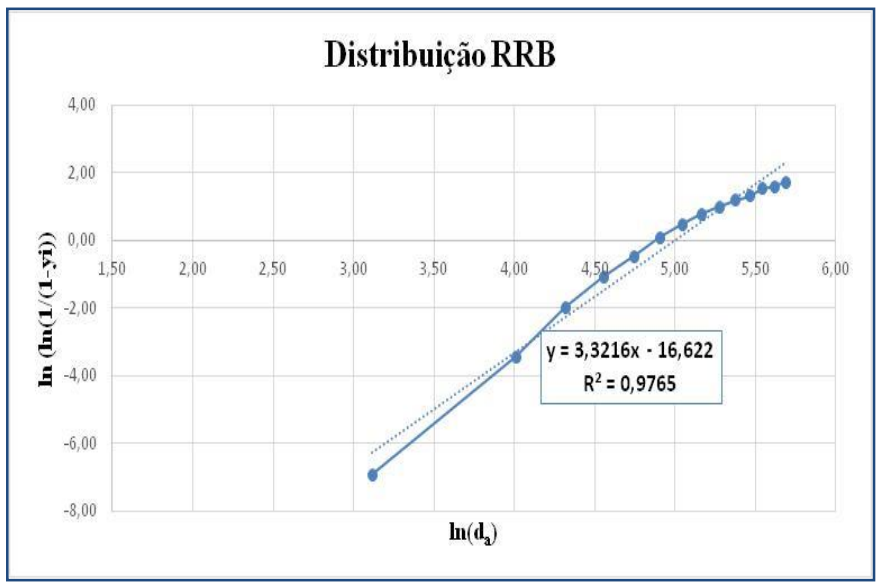

Pesagem: Em balança semi-analítica com capacidade de 2000g e precisão de centésimo de grama foram pesadas as amostras e, em seguida, conduzidos os experimentos com composições a seguir dos dois materiais, a fim de observar a eficiência do protótipo. Massas utilizadas 20, 40, 60, 80 e 100 gramas, cada peso preparado com as composições de $10 \%$, $30 \%, 50 \%, 70 \%$ e $90 \%$ de HIPS, para os experimentos com 50\% de HIPS foram conduzidos em duplicata, totalizando 30 bateladas.

\subsection{Separação da Mistura}

Separação em Protótipo: A Figura 5 mostra o protótipo em perspectiva e um corte vertical, evidenciando as tubulações e conexões que conduzem a água de um tanque para o outro.

(a)

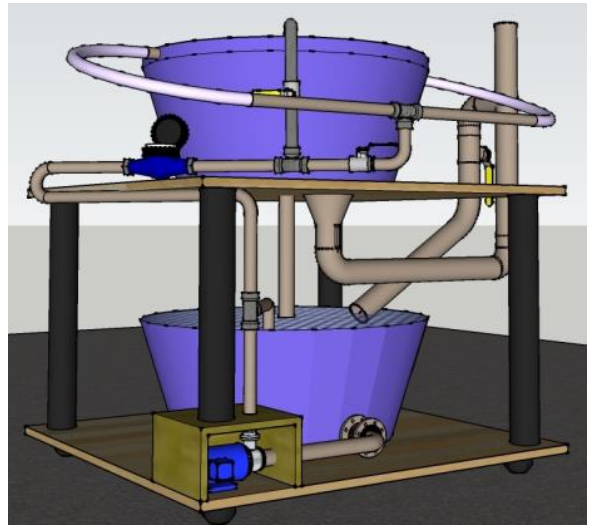

(b)

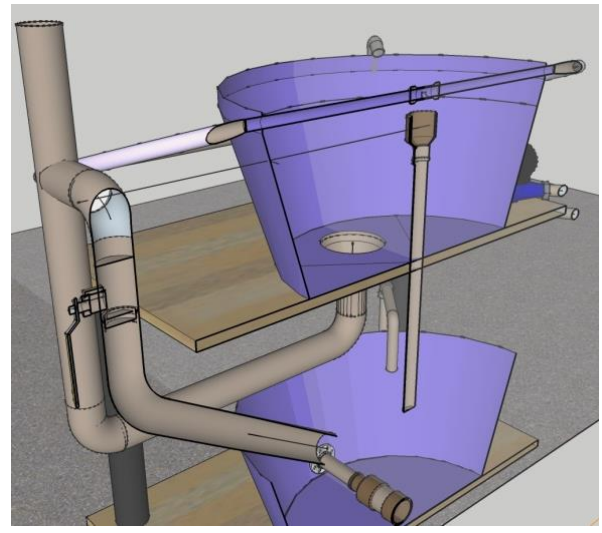

Figura 5 - Protótipo: (a) perspectiva e (b) corte vertical 
Secagem: Após a separação, as amostras eram secas em estufa durante 1 a 3 horas em temperaturas de no máximo $80^{\circ} \mathrm{C}$.

Separação Manual: as amostras secas eram submetidas a um processo de separação manual e visual por cor, para posterior pesagem e determinação da proporção de PP e HIPS. O material em menor proporção na mistura é comumente referido por contaminação. Os resultados foram registrados da seguinte forma: massa separada de PP e HIPS, massa da contaminação de PP no produto de fundo e de HIPS no de topo.

\section{Resultados - Determinação da Eficiência de Separação}

O protótipo de 100L obteve ótimos resultados em comparação aos modelos anteriores de $20 \mathrm{~L}$ e $40 \mathrm{~L}$, não apresentando ou reduzindo a incidência de falhas operacionais e automação. No entanto, mesmo com dimensões maiores e adaptações feitas, o equipamento apresentou entupimento na válvula de controle de fluxo do produto de fundo em dois do testes realizados, $70 \mathrm{~g} / 30 \mathrm{~g}$ e $90 \mathrm{~g} / 10 \mathrm{~g}$ de $\mathrm{HIPS} / \mathrm{PP}$.

Nela, são apresentados os valores da massa encontrada no fundo e topo e da massa média recuperada nessas duas frações, ou seja, quanto de HIPS recuperou-se no produto de fundo e quanto PP recuperou-se no produto de topo. Com isso, determinou-se a recuperação de cada amostra pela divisão da massa de flakes obtidos de PP ou HIPS pela massa total desse material na amostra inicial. E determinou-se também a pureza de PP e de HIPS pela divisão da massa desses materiais recuperados pelas massas total do produto topo ou fundo, respectivamente.

Tabela 3 - Resultados Experimentais da Separação. (a) Produto de Fundo e (b) Produto de Topo

\begin{tabular}{|c|c|c|c|c|}
\hline \multicolumn{5}{|c|}{ Análise do Produto de Fundo } \\
\hline $\begin{array}{c}\text { Massa } \\
\text { Mistura } \\
\text { Inicial (g) }\end{array}$ & $\begin{array}{c}\text { Massa } \\
\text { Produto de } \\
\text { Fundo }(\mathrm{g})\end{array}$ & $\begin{array}{c}\text { Massa } \\
\text { Recuperada } \\
\text { de HIPS (g) }\end{array}$ & $\begin{array}{c}\text { Recupe ração } \\
\text { HIPS (\%) }\end{array}$ & $\begin{array}{c}\text { Pureza } \\
\text { HIPS }(\%)\end{array}$ \\
\hline \multicolumn{5}{|c|}{ HIPS na Mistura Inicial: $10 \%$} \\
\hline 20 & 2,42 & 1,90 & $95,00 \%$ & $78,51 \%$ \\
\hline 40 & 6,20 & 3,35 & $83,75 \%$ & $54,03 \%$ \\
\hline 60 & 8,36 & 5,77 & $96,17 \%$ & $69,02 \%$ \\
\hline 80 & 7,20 & 6,59 & $82,38 \%$ & $91,53 \%$ \\
\hline \multirow[t]{2}{*}{100} & 6,25 & 5,33 & $53,30 \%$ & $85,28 \%$ \\
\hline & & Subtotal: & $82,12 \%$ & $75,67 \%$ \\
\hline \multicolumn{5}{|c|}{ HIPS na Mistura Inicial: $30 \%$} \\
\hline 20 & 4,37 & 4,30 & $71,67 \%$ & $98,40 \%$ \\
\hline 40 & 11,02 & 10,06 & $83,79 \%$ & $91,24 \%$ \\
\hline 60 & 14,11 & 13,91 & $77,28 \%$ & $98,58 \%$ \\
\hline 80 & 17,99 & 16,90 & $70,42 \%$ & $93,94 \%$ \\
\hline \multirow[t]{2}{*}{100} & 22,93 & 22,30 & $74,33 \%$ & $97,25 \%$ \\
\hline & & Subtotal: & $\mathbf{7 5 , 5 0 \%}$ & $\mathbf{9 5 , 8 8 \%}$ \\
\hline \multicolumn{5}{|c|}{ HIPS na Mistura Inicial: 50\% } \\
\hline 20 & 8,09 & 7,83 & $78,25 \%$ & $96,78 \%$ \\
\hline 40 & 16,54 & 15,35 & $76,75 \%$ & $92,81 \%$ \\
\hline 60 & 27,69 & 25,86 & $86,20 \%$ & $93,41 \%$ \\
\hline 80 & 35,55 & 34,08 & $85,20 \%$ & $95,88 \%$ \\
\hline \multirow[t]{2}{*}{100} & 23,82 & 23,29 & $46,57 \%$ & $97,76 \%$ \\
\hline & & Subtotal: & $\mathbf{7 4 , 5 9 \%}$ & $95,33 \%$ \\
\hline \multicolumn{5}{|c|}{ HIPS na Mistura Inicial: 70\% } \\
\hline 20 & 9,51 & 9,44 & $67,43 \%$ & $99,26 \%$ \\
\hline 40 & 12,26 & 12,11 & $43,25 \%$ & $98,78 \%$ \\
\hline 60 & 37,07 & 36,70 & $87,38 \%$ & $99,00 \%$ \\
\hline \multirow[t]{2}{*}{80} & 46,56 & 46,43 & $82,91 \%$ & $99,72 \%$ \\
\hline & & Subtotal: & $70,24 \%$ & $99,19 \%$ \\
\hline \multicolumn{5}{|c|}{ HIPS na Mistura Inicial: $90 \%$} \\
\hline 20 & 16,74 & 16,21 & $90,06 \%$ & $96,83 \%$ \\
\hline 40 & 35,76 & 35,36 & $98,22 \%$ & $98,88 \%$ \\
\hline 60 & 50,84 & 49,82 & $92,26 \%$ & $97,99 \%$ \\
\hline \multirow[t]{3}{*}{80} & 60,33 & 58,65 & $81,46 \%$ & $97,22 \%$ \\
\hline & & Subtotal: & $90,50 \%$ & $\mathbf{9 7 , 7 3 \%}$ \\
\hline & & & $78,44 \%$ & $92,27 \%$ \\
\hline
\end{tabular}

\begin{tabular}{|c|c|c|c|c|}
\hline \multicolumn{5}{|c|}{ Análise do Produto de Topo } \\
\hline $\begin{array}{c}\text { Massa } \\
\text { Mistura } \\
\text { Inicial (g) }\end{array}$ & $\begin{array}{l}\text { Massa } \\
\text { Produto de } \\
\text { Topo }(g)\end{array}$ & $\begin{array}{c}\text { Massa } \\
\text { Recuperada } \\
\text { de PP (g) }\end{array}$ & $\begin{array}{c}\text { Recuperação } \\
\text { PP }(\%)\end{array}$ & $\begin{array}{c}\text { Pureza PP } \\
(\%)\end{array}$ \\
\hline \multicolumn{5}{|c|}{ PP na Mistura Inicial: 90\% } \\
\hline 20 & 17,58 & 17,23 & $95,72 \%$ & $98,01 \%$ \\
\hline 40 & 33,76 & 33,15 & $92,08 \%$ & $98,19 \%$ \\
\hline 60 & 51,54 & 50,93 & $94,31 \%$ & $98,82 \%$ \\
\hline 80 & 72,72 & 70,61 & $98,07 \%$ & $97,10 \%$ \\
\hline 100 & 93,66 & 88,61 & $98,46 \%$ & $94,61 \%$ \\
\hline & & Subtotal: & $\mathbf{9 5 , 7 3 \%}$ & $97,35 \%$ \\
\hline \multicolumn{5}{|c|}{ PP na Mistura Inicial: $70 \%$} \\
\hline 20 & 16,36 & 14,02 & $100,14 \%$ & $85,70 \%$ \\
\hline 40 & 28,76 & 26,93 & $96,18 \%$ & $93,64 \%$ \\
\hline 60 & 45,82 & 41,46 & $98,71 \%$ & $90,48 \%$ \\
\hline 80 & 61,52 & 53,75 & $95,98 \%$ & $87,37 \%$ \\
\hline 100 & 73,76 & 65,33 & $93,33 \%$ & $88,57 \%$ \\
\hline & & Subtotal: & $96,87 \%$ & $89,15 \%$ \\
\hline \multicolumn{5}{|c|}{ PP na Mistura Inicial: 50\% } \\
\hline 20 & 10,89 & 8,70 & $87,00 \%$ & $79,89 \%$ \\
\hline 40 & 22,81 & 17,79 & $88,95 \%$ & $77,99 \%$ \\
\hline 60 & 32,79 & 27,22 & $90,72 \%$ & $83,01 \%$ \\
\hline 80 & 43,85 & 36,67 & $91,66 \%$ & $83,61 \%$ \\
\hline 100 & 75,58 & 49,91 & $99,83 \%$ & $66,04 \%$ \\
\hline & & Subtotal: & $91,63 \%$ & $78,11 \%$ \\
\hline \multicolumn{5}{|c|}{ PP na Mistura Inicial: 30\% } \\
\hline 20 & 10,60 & 6,09 & $101,50 \%$ & $57,45 \%$ \\
\hline 40 & 27,71 & 12,03 & $100,25 \%$ & $43,41 \%$ \\
\hline 60 & 22,80 & 17,76 & $98,67 \%$ & $77,89 \%$ \\
\hline 80 & 34,04 & 24,82 & $103,42 \%$ & $72,91 \%$ \\
\hline & & Subtotal: & $100,96 \%$ & $62,92 \%$ \\
\hline \multicolumn{5}{|c|}{ PP na Mistura Inicial: 10\% } \\
\hline 20 & 3,16 & 1,50 & $75,00 \%$ & $47,47 \%$ \\
\hline 40 & 4,21 & 3,57 & $89,25 \%$ & $84,80 \%$ \\
\hline 60 & 9,28 & 5,10 & $85,00 \%$ & $54,96 \%$ \\
\hline \multirow[t]{2}{*}{80} & 18,76 & 6,52 & $81,50 \%$ & $34,75 \%$ \\
\hline & & Subtotal: & $82,69 \%$ & $55,49 \%$ \\
\hline & & & $93,73 \%$ & $78,12 \%$ \\
\hline
\end{tabular}




\section{CONCLUSÕES}

A partir dos resultados apresentados na tabela 3, conclui-se que é possível separar frações de PP e de HIPS pelo método proposto com o equipamento desenvolvido de modo eficiente.

O funcionamento final do protótipo desenvolvido operou em sistema de batelada, possui fácil operação e sua estrutura permite a integração em série para aumento da qualidade do produto final. O protótipo apresentou baixo custo operacional e utilizou apenas água pura como meio de separação, facilitando o descarte de efluentes gerados. Pode ainda contar com a separação de outros plásticos, além dos já descritos, com base na diferença de densidade.

A técnica para determinação de tamanho de partículas utilizada com auxilio do programa ImageJ e câmera fotográfica, somada ao fato da distribuição de tamanhos de partículas obedecer o modelo RRB irá, em experimentos futuros, proporcionar de forma prática uma análise da influência do tamanho de partículas ao ser aplicada aos flakes dos produtos de topo e fundo.

Embora o estudo feito seja bastante considerável em termos experimentais, são necessários outros estudos com outras configurações estruturais. Além disso, a execução em bateladas em séries ou a transformação do processo em contínuo são alternativas interessantes para obtenção de um produto mais puro.

\section{REFERÊNCIAS}

AL-SALEM, S. M., LETTIERI, P., BAEYENS, J. Recycling and recovery routes of plastic solid waste (PSW): A review. Waste Management, n. 29, p. 2625-2643, 2009.

DE OLIVEIRA, M. C. B. R., Gestão de Resíduos Plásticos Pós-Consumo: Perspectivas para a Reciclagem no Brasil, Dissertação de M.Sc., UFRJ/COPPE, Rio de Janeiro, RJ, Brasil, 2012.

Freeware ImageJ, disponível em http://rsbweb.nih.gov/. Acesso em janeiro de 2014.

GENT, M. R.; MENENDEZ, M.; TORAÑO, J.; DIEGO, I. Recycling of plastic waste by density separation: prospects for optimization. Waste Management \& Research, v. 27, p. 175187, 2009.

LEI FEDERAL No 12.305. Institui a Política Nacional de Resíduos Sólidos (PNRS), Brasil, 02 de agosto de 2010.

PLASTICS EUROPE, 2013. Plastics - the Facts 2013. An analysis of European latest plastics production, demand and waste data. Disponível em: < http://www.plasticseurope.com >. Acesso em: 20 de janeiro de 2014.

PONGSTABODEE, S.; KUNACHITPIMOL, N.; DAMRONGLERD, S. Combination of three-stage sink-float method and selective flotation technique for separation of mixed postconsumer plastic waste. Waste Management, v. 28, p. 475-483, 2008. 